Paula Salgado Lucena Rodrigues

\title{
Um Sistema de Geração de Expressões Faciais Dinâmicas em Animações Faciais 3D com Processamento de Fala
}

Tese apresentada ao Programa de Pós-graduação em Informática do Departamento de Informática da PUC-Rio como requisito parcial para obtenção do título de Doutor em Informática

Orientador

Prof. Bruno Feijó

Co-Orientador: Prof. Luiz Carlos P.R. Velho 


\title{
Paula Salgado Lucena Rodrigues
}

\section{Um Sistema de Geração de Expressões Faciais Dinâmicas em Animações Faciais 3D com \\ Processamento de Fala}

Tese apresentada ao Programa de Pós-graduação em Informática do Departamento de Informática do Centro Técnico Científico da PUC-Rio como requisito parcial para obtenção do título de Doutor em Informática. Aprovada pela Comissão Examinadora abaixo assinada.

\author{
Prof. Bruno Feijó \\ Orientador \\ Departamento de Informática - PUC-Rio \\ Prof. Luiz Carlos P.R. Velho \\ Co-Orientador \\ IMPA \\ Prof. Antonio L. Furtado \\ Departamento de Informática, PUC-Rio \\ Prof. Waldemar Celes \\ Departamento de Informática, PUC-Rio
}

Prof. Soraia Raupp Musse

Faculdade de Informática, PUCRS

Prof. Mônica Maria Ferreira da Costa

MidiArte

Prof. Lisandro Lovisolo

UERJ

Prof. José Eugênio Leal

Coordenador Setorial do Centro Técnico Científico - PUC-Rio 
Todos os direitos reservados. É proibida a reprodução total ou parcial do trabalho sem autorização da universidade, do autor e do orientador.

\section{Paula Salgado Lucena Rodrigues}

Graduou-se como Bacharel em Ciência da Computação pela UFPE (Universidade Federal de Pernambuco) em 1999. Obteve em 2002 o Mestrado em Informática pela PUC-Rio (Pontifícia Universidade Católica do Rio de Janeiro). Ao longo do Doutorado atuou como pesquisadora dos Laboratórios TeleMídia, VisionLab e TecGraf, do Departamento de Informática da PUC-Rio, e ministra aulas no curso de Graduação em Engenharia dessa mesma instituição desde 2005. Ao longo desses anos atuou como professora em cursos para empresas como a Petrobras (2003-2005) e em cursos de Especialização no CCE PUC-Rio (2002-2003) e na Fejal CESMAC (2004-até o momento).

Ficha Catalográfica

Rodrigues, Paula Salgado Lucena

Um Sistema de Geração de Expressões Faciais Dinâmicas em Animações Faciais 3D com Processamento de Fala / Paula Salgado Lucena Rodrigues; orientador: Bruno Feijó; coorientador: Luiz Carlos P.R. Velho. - Rio de Janeiro : PUCRio, Departamento de Informática, 2007.

v., 161 f: il. ; $29,7 \mathrm{~cm}$

1. Tese (doutorado) - Pontifícia Universidade Católica do Rio de Janeiro, Departamento de Informática.

Inclui referências bibliográficas.

1. Informática - Tese. 2. Animação Facial. 3. Expressões Faciais Dinâmicas. 4. Processamento de Fala. 5. Modelo de Emoção. 6. Hipercubo Emocional. 7. Personagens Virtuais. 8. Personagens Virtuais Falantes. 9. MPEG-4. I. Feijó, Bruno. II. Velho, Luiz. III. Pontifícia Universidade Católica do Rio de Janeiro. Departamento de Informática. IV. Título. 
Aos nossos filhos Milton e Maria Clara que viveram intensamente esta Tese durante as suas gestações. Por eles, eu busquei garra e esperança de terminar este trabalho. 


\section{Agradecimentos}

A Deus pela vida, saúde, sabedoria, esperança e perseverança.

À minha irmã Cynthia por ser meu exemplo e inspiração para não desistir dos sonhos. Agradeço pela vida, pelo amor, pela amizade, pela cumplicidade, pela doação e dedicação. Mesma na distância física, ela está sempre presente na minha vida.

Ao meu marido Rogério por ser amigo, companheiro, cúmplice, ... por me fazer feliz. Esta Tese é resultado de um sonho nosso, onde ele se sacrificou junto comigo, confiando e apostando nesta vitória.

À Asla Sá pela amizade verdadeira que construímos. Agradeço também por todas as nossas discussões matemáticas, pelas idéias e concretizações. Devo a ela muito do resultado deste trabalho!

Aos meus pais Paulo e Maria de Lourdes por tudo que tenho e que sou. Pelos ensinamentos como ser humano, por acreditarem nos meus sonhos, por serem meus melhores amigos e companheiros.

Ao professor Luiz Velho, exemplo de profissional e de ser humano. Agradeço a ele por acreditar na minha "animação", desde o Mestrado, por sonhar junto comigo, por me fazer persistir neste sonho. Agradeço também por todo ensinamento, profissional e pessoal, sendo um exemplo a ser seguido.

Ao professor Bruno Feijó pela confiança e pelas oportunidades propiciadas.

À minha família e a família de Rogério por respeitarem o "momento Tese", por toda torcida, paciência, orações e pensamento positivo.

As minhas "irmã cariocas" Christiane Caula, Liliane Thompson, Luciana Lima, Lucimar Martins, Maíra Greco, Taciana Melcop e Viviane Braconi pela amizade, companheirismo, torcida, cumplicidade... por serem minha família aqui no Rio.

Ao professor Luiz Fernando Gomes Soares pela oportunidade de trabalhar no TeleMídia e no projeto de TV Digital. Agradeço também aos amigos do Laboratório Telemídia, incluindo Lorenza Moreno e Suzana Maranhão.

Aos professores Antonio Furtado e Soraia Raup pelo trabalho no projeto Virtus.

Aos professores Marcelo Gattass e Waldemar Celes pela oportunidade de retornar ao TecGraf que foi de grande incentivo para a conclusão desta Tese.

Aos amigos do TecGraf, principalmente, ao Grupo de Visualização pela torcida, incentivo e inspirações diários.

Aos amigos de Recife, Sérgio Soares, Isabella Aragão, Maria Luisa Ribeiro, Conceição e Breno Sayão, Patricia Ramos, por serem minha fortaleza e me passarem a certeza que estamos sempre juntos, mesmo na distância física. 
Ao $\mathrm{CNPq}$ e à PUC-Rio, pelos auxílios concedidos, sem os quais este trabalho não poderia ter sido realizado.

Aos meus professores do Departamento de Informática e do IMPA. Com o conhecimento dado por eles consegui crescer profissionalmente e realizar meus sonhos acadêmicos.

Ao pessoal do Departamento de Informática pela ajuda de todos os dias, em particular à Fatima, Emmanuelle e ao Alex. 


\section{Resumo}

Rodrigues, Paula Salgado Lucena; Feijó, Bruno; Velho, Luiz. Um Sistema de Geração de Expressões Faciais Dinâmicas em Animações Faciais 3D com Processamento de Fala. Rio de Janeiro, 2007. 161p. Tese de Doutorado - Departamento de Informática, Pontifícia Universidade Católica do Rio de Janeiro.

Esta tese apresenta um sistema para geração de expressões faciais dinâmicas sincronizadas com a fala em uma face realista tridimensional. Entende-se por expressões faciais dinâmicas aquelas que variam ao longo do tempo e que semanticamente estão relacionadas às emoções, à fala e a fenômenos afetivos que podem modificar o comportamento de uma face em uma animação. A tese define um modelo de emoção para personagens virtuais falantes, denominado VeeM (Virtual emotion-to-expression Model), proposto a partir de uma releitura e uma reestruturação do modelo do círculo emocional de Plutchik. O VeeM introduz o conceito de um hipercubo emocional no espaço canônico do $R^{4}$ para combinar emoções básicas, dando origem a emoções derivadas. Para validação do $V e e M$ é desenvolvida uma ferramenta de autoria e apresentação de animações faciais denominada DynaFeX (Dynamic Facial eXpression), onde um processamento de fala é realizado para permitir o sincronismo entre fonemas e visemas. A ferramenta permite a definição e o refinamento de emoções para cada quadro ou grupo de quadros de uma animação facial. O subsistema de autoria permite também, alternativamente, uma manipulação em alto-nível, através de scripts de animação. O subsistema de apresentação controla de modo sincronizado a fala da personagem e os aspectos emocionais editados. A DynaFeX faz uso de uma malha poligonal tridimensional baseada no padrão MPEG-4 de animação facial, favorecendo a interoperabilidade da ferramenta com outros sistemas de animação facial.

\section{Palavras-chave}

Animação Facial. Expressões Faciais Dinâmicas. Processamento de Fala. Modelo de Emoção. Hipercubo Emocional. Personagens Virtuais. Personagens Virtuais Falantes. MPEG-4. 


\section{Abstract}

Rodrigues, Paula Salgado Lucena; Feijó, Bruno; Velho, Luiz. A System for Generating Dynamic Facial Expressions in 3D Facial Animation with Speech Processing. Rio de Janeiro, 2007. 161p. PhD Thesis - Department of Informática, Pontifícia Universidade Católica do Rio de Janeiro.

This thesis presents a system for generating dynamic facial expressions synchronized with speech, rendered using a tridimensional realistic face. Dynamic facial expressions are those temporal-based facial expressions semantically related with emotions, speech and affective inputs that can modify a facial animation behavior. The thesis defines an emotion model for speech virtual actors, named VeeM (Virtual emotion-to-expression Model), which is based on a revision of the emotional wheel of Plutchik model. The VeeM introduces the emotional hypercube concept in the $R^{4}$ canonical space to combine pure emotions and create new derived emotions. In order to validate $V e e M$, it has been developed an authoring and player facial animation tool, named DynaFeX (Dynamic Facial eXpression), where a speech processing is realized to allow the phoneme and viseme synchronization. The tool allows either the definition and refinement of emotions for each frame, or group of frames, as the facial animation edition using a high-level approach based on animation scripts. The tool player controls the animation presentation synchronizing the speech and emotional features with the virtual character performance. DynaFeX is built over a tridimensional polygonal mesh, compliant with MPEG-4 facial animation standard, what favors tool interoperability with other facial animation systems.

\section{Keywords}

Facial Animation. Dynamic Facial Expressions. Speech Processing. Emotion Model. Emotional Hypercube. Virtual Characters. Virtual Talking Characters. MPEG-4. 


\section{Sumário}

$\begin{array}{lll}1 & \text { Introdução } & \mathbf{1 5}\end{array}$

\begin{tabular}{lll}
\hline 1.1 & Estado da Arte em Animação Facial & 15
\end{tabular}

$\begin{array}{lll}1.2 & \text { Desafios da Área } & 18\end{array}$

1.3 Parametrização Ideal para Sistemas de Animação Facial 19

$\begin{array}{lll}1.4 & \text { Contribuições Esperadas } & 21\end{array}$

1.5 Estrutura do Documento 22

2 Espaços de Emoção $\quad 24$

2.1 Estado da Arte para os Modelos de Emoção 24

$\begin{array}{lll}2.2 & \text { Emoções Básicas } & 26\end{array}$

2.3 Emoções na Teoria da Avaliação 28

$\begin{array}{lll}2.4 & \text { Modelo de Humor } & 30\end{array}$

2.5 Modelos de Emoção em Sistemas Computacionais 32

3 VeeM: Um Modelo de Emoção para Personagens Virtuais 35

3.1 Esquema de Classificação de Emoção em Eixos Bipolares 36

\begin{tabular}{|lll}
\hline 3.2 & Combinação de Emoções Puras para Geração de & 37
\end{tabular}

3.3 O Hipercubo Emocional para o VeeM 39

3.4 Taxonomia das Emoções Derivadas a partir da

3.5 Incorporação de Fenômenos Afetivos e Expressões

3.6 Visualização da Emoção: Expressão Facial 52

\begin{tabular}{ll|}
\hline 3.7 & Análise do VeeM e seu Hipercubo Emocional \\
& segundo a Parametrização Ideal para Animação Facial
\end{tabular}

$4 \quad$ Espaços de Expressões Faciais no Padrão MPEG-4

$4.1 \quad$ Espaço de Expressões Faciais 56

4.2 Apresentação do Padrão MPEG-4 57

4.3 Especificação da Face no Padrão MPEG-4 58

4.4 Expressões Faciais no Padrão MPEG-4 62

4.5 Visema e Lip-Sync no MPEG-4 64

\begin{tabular}{lll}
\hline 4.6 & Especificação de um Modelo de Face no & 66
\end{tabular}

4.7 Análise do Padrão MPEG-4 segundo a Parametrização Ideal para Animação Facial 67

5 DynaFeX: Uma Ferramenta de Edição e Exibição

$\begin{array}{lll}5.1 & \text { Visão Geral da DynaFeX } & 71\end{array}$

$\begin{array}{lll}5.2 & \text { Fornecimento de Arquivos de Entrada } & 74\end{array}$

$\begin{array}{lll}5.3 & \text { Geração da Estrutura Fonética da Fala } & 77\end{array}$ 
5.4 Definição e Refinamento de Emoções para

Geração de Expressões Faciais 82

5.5 Combinação dos FAPs 85

5.6 Execução da Animação Facial com Sincronismo

5.7 Implementação dos Fenômenos Afetivos e das Expressões Não-Verbais 89

$\begin{array}{lll}5.8 \text { Estudos de Casos } & 92\end{array}$

\begin{tabular}{lll}
\hline 6 & Trabalhos Relacionados & 97
\end{tabular}

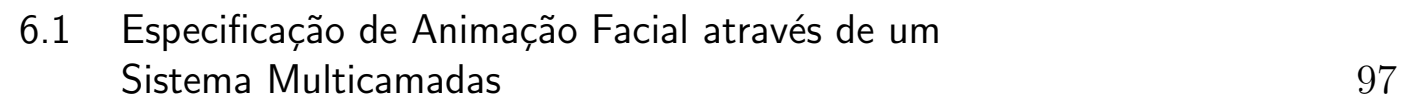

\begin{tabular}{|l|l|}
6.2 Síntese e Edição de Animação Facial Expressiva com \\
Controle através de Isomapas de Fonemas
\end{tabular}

$\begin{array}{lll}6.3 \text { Modificadores Paramétricos Qualitativos em um } & 101 \\ \text { Sistema de Animação Facial } & \end{array}$

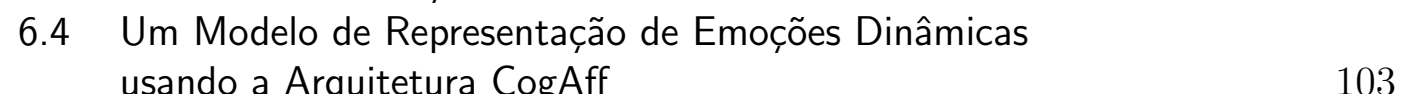

6.5 Síntese de Outros Trabalhos Relevantes 105

$\begin{array}{lll}6.6 & \text { Análise Comparativa } & 108\end{array}$

$\begin{array}{lll}7 & \text { Conclusão } & 110\end{array}$

$\begin{array}{lll}7.1 & \text { Contribuições da Tese } & 111\end{array}$

$\begin{array}{lll}7.2 & \text { Trabalhos Futuros } & 113\end{array}$

\begin{tabular}{lr}
\hline Referências Bibliográficas & 115
\end{tabular}

\begin{tabular}{lr}
\hline Sumário das notações & 126
\end{tabular}

\begin{tabular}{lll}
\hline B & Especificação dos Parâmetros da Animação Facial & \\
\hline no Padrão MPEG-4 & 138
\end{tabular}

C Especificação dos FAPs na DynaFeX para as

Expressões Faciais dos Visemas e das Emoções

Puras a partir do Hipercubo Emocional 


\section{Lista de figuras}

1.1 Taxonomia da Animação Facial. 16

2.1 Emoções universais definidas por Ekman. 27

$\begin{array}{lll}2.2 & \text { Emoções básicas definidas por Plutchik. } & 27\end{array}$

2.3 Caricaturas para emoções puras definidas por Plutchik. 28

2.4 As três camadas da arquitetura CogAff. 29

2.5 Interface gráfica do sistema EmoSpeak. 33

3.1 Visualização completa do círculo de Plutchik. 37

3.2 Configuração para emoções opostas. 38

3.3 Combinações de emoções. 38

\begin{tabular}{lll}
\hline 3.4 & Emoções básicas nos eixos do VeeM. & 39
\end{tabular}

3.5 Emoções do plano $x y$. 43

3.6 Emoções do plano $x z$. 44

3.7 Emoções do plano xw. 45

3.8 Emoções do plano yz. 46

3.9 Emoções do plano yw.

3.10 Emoções do plano zw. 49

3.11 Esquematização do VeeM. 50

4.1 Músculos das expressões faciais.

4.2 A face neutra e definição das FAPUs. $\quad 59$

4.3 Pontos característicos do padrão MPEG-4.

4.4 Exemplo da animação de um FAP. 62

4.5 Expressões faciais no MPEG-4. 63

4.6 Visemas para expressão facial feliz. $\quad 66$

5.1 Visão geral da ferramenta DynaFeX.

5.2 Padrão de projeto aplicado na DynaFeX.

5.3 Exemplo de descrição textual e de emoção. $\quad 76$

5.4 Exemplo de arquivo fap. 76

5.5 Módulo de estrutura fonética da fala. $\quad 77$

5.6 Arquivos de saída do reconhecedor de fala. 78

5.7 Expressão facial dos 15 visemas da DynaFeX.

5.8 Expressões puras do VeeM na Dynafex. 82

5.9 Edição alto-nível. 83

5.10 Edição baixo-nível.

5.11 Módulo de combinação de FAPs.

5.12 Interface gráfica para movimento dos olhos. 90

5.13 Configuração dos olhos para algumas emoções. 91

5.14 Análise comparativa do movimento dos olhos. 92

5.15 Integração das ferramentas ao formatador HyperProp. 94

5.16 Exemplo de execução. 94

5.17 Arquitetura da junção do ETHs com o LOGTELL. 95

5.18 Cena renderizada no ambiente de narração. 96 
6.1 As cinco camadas do sistema SMILE. 98

6.2 Visão geral do sistema eFASE. $\quad 99$

\begin{tabular}{lll}
\hline 6.3 & Arquitetura do sistema EE-FAS. & 104
\end{tabular}

6.4 Definição das categorias de emoções. 106

6.5 Disco emocional para combinação de emoções. 107

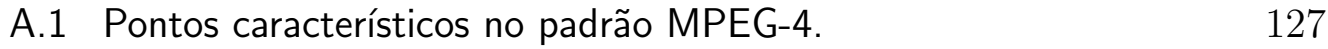

C.1 Expressão facial dos 15 visemas na DynaFeX. 151

\begin{tabular}{lll}
\hline C.2 & Emoções puras na DynaFeX. & 157
\end{tabular}

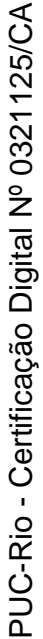




\section{Lista de tabelas}

2.1 Emoções Básicas 34

3.1 Semântica das emoções puras. 40

4.1 Grupo de FAPs 61

4.2 Expressões faciais primárias 64

4.3 Visemas e os fonemas relacionados. 65

5.1 Mapeamento dos fonemas para visemas. $\quad 80$

6.1 Parâmetros e elementos do Effort. 102

C.1 Intervalo sugerido dos FAPs nos eixos $x$ e $y$. 160

C.2 Intervalo sugerido dos FAPs nos eixos $z$ e $w$. 161 
"Animation is not the art of drawings that move, but rather the art of movements that are drawn. What happens between each frame is more important than what happens in each frame. Animation is the art of manipulating the invisible interstices that lie between frames."

Norman McLaren, Chuck Jones: Life of Animated Cartoonist. 